\title{
Metástase orbitária como único achado em paciente com hepatocarcinoma: relato de caso
}

\author{
An unusual orbital metastatic lesion: the only finding in a case \\ of hepatocellular carcinoma: case report
}

\author{
Nilson Lopes da Fonseca Júnior ${ }^{1}$ \\ Luciana Frizon² \\ Luis Paves ${ }^{3}$ \\ Angela Maria Borri Wolosker ${ }^{4}$ \\ PauloGóis Manso 5
}

\begin{tabular}{|l|}
\hline RESUMO \\
\hline Metástases orbitárias de hepatocarcinoma (HCC) são raras. Os autores têm \\
o objetivo de relatar o caso de um paciente que apresentou metástase \\
orbitária como único achado de um HCC. Paciente masculino, 57 anos, \\
apresentando massa em região temporal direita, associada à proptose, dor \\
e hiperemia. Os exames de imagem revelaram volumosa massa em região \\
temporal, estendendo-se para fossa temporal com destruição da parede \\
orbitária e extensão intracraniana. A tomografia computadorizada de \\
abdome demonstrou tumor primário no fígado. Foi realizada biópsia \\
incisional através de uma orbitotomia anterior cujo estudo anátomo- \\
patológico diagnosticou lesão metastática de hepatocarcinoma. O estudo \\
imuno-histoquímico com marcador para CAM 5.2 e CEA foi positivo. A \\
avaliação sistêmica não revelou outras lesões. O tumor evoluiu com \\
rápido crescimento com óbito 15 meses após o diagnóstico. Metástases \\
orbitárias do carcinoma hepatocelular são raras. Nosso caso foi relevante \\
não só pela raridade desta lesão orbitária, como também pela ausência de \\
outras lesões metastáticas e de sintomas sistêmicos.
\end{tabular}

Descritores: Metástase neoplásica; Neoplasias orbitárias/secundário; Carcinoma hepatocelular; Neoplasias hepáticas [Tipo de publicação]

\section{INTRODUÇÃO}

O hepatocarcinoma (HCC), também conhecido como hepatoma, é a neoplasia maligna originada das células do parênquima hepático. Relativamente raro, corresponde a $2 \%$ de todos os processos malignos nos Estados Unidos e na Europa Ocidental. Entretanto, a OMS aponta o hepatocarcinoma como a segunda causa de óbito por câncer em seres humanos, pela alta incidência em regiões da África e Pacífico Oeste ${ }^{(1-3)}$.

O hepatoma é predominantemente uma doença do sexo masculino, com origem habitual em um fígado cirrótico ${ }^{(1-2)}$. Os pacientes portadores de HCC raramente apresentam lesões metastáticas orbitárias, sendo mais freqüentes a invasão e obstrução das veias porta e hepáticas e metástases para gânglios linfáticos regionais e pulmões ${ }^{(1-4)}$.

As lesões metastáticas representam cerca de $2 \%$ de todas as doenças orbitárias e 10 a $12 \%$ das lesões malignas da órbita, sendo a mama o sítio primário mais comum (42\%).

Quanto ao surgimento destas lesões metastáticas, cerca de 30\% dos casos são concomitantes ao aparecimento da lesão primária, 42\% surgem tardiamente e $35 \%$ dos pacientes não apresentam diagnóstico do tumor primário, ao manifestar a lesão orbitária. 
O objetivo deste trabalho é relatar o quadro clínico, radiológico e histopatológico de um paciente que apresentou uma tumoração orbitária como primeira manifestação de um hepatocarcinoma.

\section{RELATO DE CASO}

Paciente do sexo masculino, 57 anos de idade foi encaminhado ao setor de Órbita do Departamento de Oftalmologia da Universidade Federal de São Paulo (UNIFESP) em janeiro de 2004 para avaliação de proptose no olho direito. Apresentava diminuição da acuidade visual no olho direito associada a dor e proptose há um mês. Como antecendentes pessoais oftalmológicos revelava facoemulsificação com implante de lente intra-ocular em ambos os olhos em 1996, e descolamento total da retina do olho esquerdo com conseqüente amaurose, após trauma ocular há 5 anos. Referia tabagismo e etilismo social por 5 anos. Negava doenças ou sintomas sistêmicos.

Ao exame oftalmológico, apresentava acuidade visual de 20/200 no olho direito e ausência de percepção luminosa no olho esquerdo. À motilidade extrínseca ocular apresentava limitação em todas as versões. A medida da exoftalmometria utilizando-se o exoftalmômetro de Hertel foi de $22 \mathrm{~mm}$ em olho direito e de $14 \mathrm{~mm}$ em olho esquerdo. À ectoscopia, o olho direito apresentava leve edema palpebral superior, discreta quemose e tumoração periocular em região temporal, e o olho esquerdo apresentava-se sem alterações. A tonometria de aplanação era de $32 \mathrm{mmHg}$ no olho direito e de $14 \mathrm{mmHg}$ no olho esquerdo. No mapeamento de retina, o olho direito apresentava retinosquisis e algumas dobras de coróide, e o olho esquerdo, descolamento total de retina.

À palpação notou-se uma massa sólida no quadrante superior da órbita direita (Figura 1A), de consistência endurecida, sem frêmito. Não foram observados linfonodos regionais palpáveis.

O exame de tomografia computadorizada (TC) de órbita (Figura 1B) e a ressonância nuclear magnética (RNM) de órbita demonstraram uma massa localizada no quadrante temporal superior da órbita direita com destruição óssea, invadindo seio frontal e espaço mastóideo, associada a extensão intracraniana e com realce heterogêneo ao contraste. $\mathrm{O}$ exame de TC de abdômen demonstrava área de alteração perfusional no segmento lateral do lobo hepático esquerdo com imagem nodular associada de $2 \mathrm{~cm} \times 2 \mathrm{~cm}$ (Figura 1C), demonstrando o sítio primário do hepatocarcinoma.

A lesão foi biopsiada através de uma orbitotomia anterior e a análise anátomo-patológica demonstrou estruturas trabeculares com citoplasma eosinofílico, bem como canalículos biliares. As células tumorais mostravam núcleos vesiculares com mitoses, fazendo diagnóstico de carcinoma hepatocelular (Figura 2A). Na imuno-histoquímica, as células tumorais apresentaram positividade para CAM 5.2 (low-molecularweight cytokeratin) e CEA (antígeno carcinoembrionário) (Figura 2B e Figura 2C).

A investigação clínica sistêmica demonstrou anemia moderada. Os exames de função hepática, sorologia para hepatite A e C, alfa-feto proteína (AFP), radiografias de ossos longos e coluna vertebral e TC de tórax apresentavam-se dentro dos padrões de normalidade. Apenas o antígeno de superfície (HBsAg) era positivo no exame sorológico para hepatite B.

O paciente optou pelo abandono do tratamento clínico e cirúrgico. Apresentou rápido crescimento tumoral em poucos meses, retornando ao serviço para consulta. Evoluiu a óbito em 15 meses.

\section{COMENTÁRIOS}

O carcinoma hepatocelular está relacionado principalmente à cirrose por infecção pelos vírus das hepatites $\mathrm{B}$ e C, bem como pela hemocromatose, cirrose alcoólica, cirrose biliar primária, doença de Wilson, exposição a aflatoxina e micotoxina derivada do Aspergillus flavus ${ }^{(1-2,5-6)}$.

Este tumor comumente metastatiza para os pulmões, cérebro e ossos. Outros locais menos freqüentes seriam o peritônio e a glândula adrenal, e raramente a cavidade nasal, órbita, bexiga, ovário, glândula parótida, calvário ${ }^{(5-8)}$.
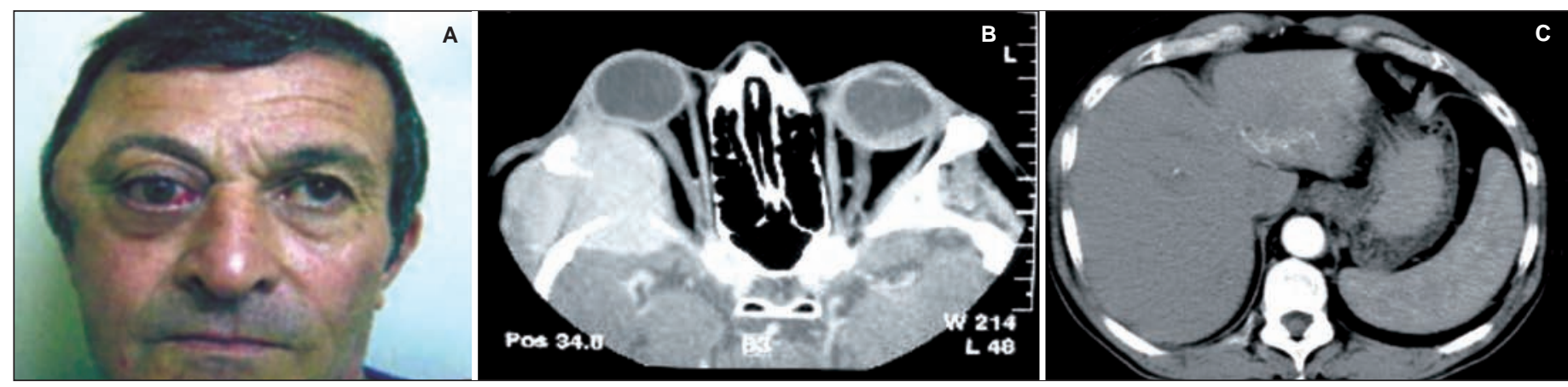

Figura 1 - A) Apresentaç̃o clínica - olho direito apresentava leve edema palpebral superior, discreta quemose e tumoração periocular em região temporal; B) Corte axial de um exame de tomografia computadorizada de órbita (janela de partes moles). Observa-se uma massa sólida comprometendo a asa maior do esfenóide à direita com extensão para fossa temporal, fossa média craniana e cavidade orbitária associada a proptose do globo ocular direito; C) Corte axial de um exame de tomografia computadorizada de abdomen (janela de partes moles): presença de lesão heterogênea de limites imprecisos no lobo hepático esquerdo. 

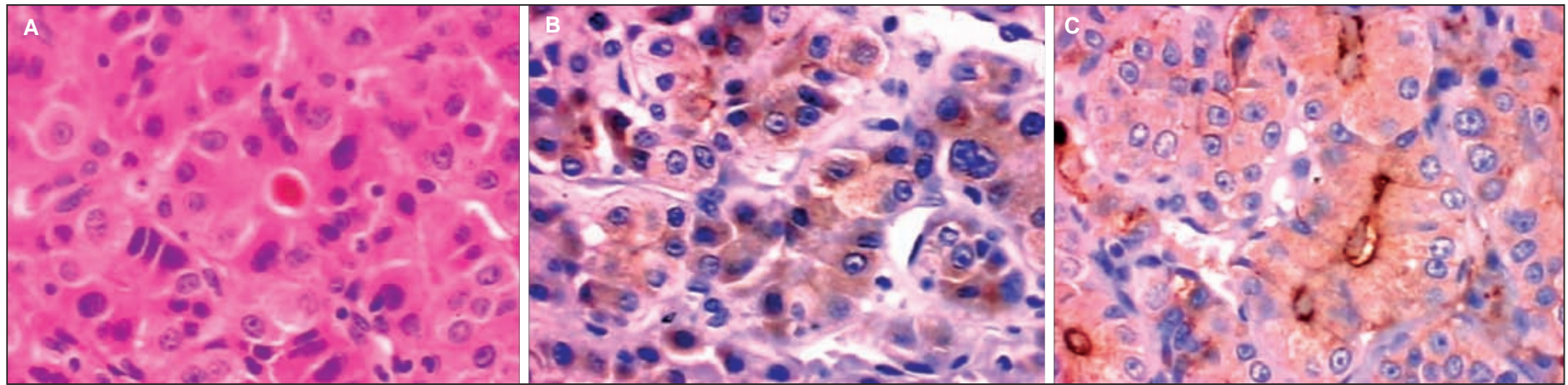

Figura 2 - Fotomicrografia - Lesão metastática de hepatocarcinoma - Aumento de 400X. A) HE. Estruturas trabeculares com citoplasma eosinofílico, bem como canalículos biliares. As células tumorais mostram núcleos vesiculares com mitoses; B) IH. CAM 5.2 - Células tumorais apresentam positividade para este marcador; C) IH. CEA - Células tumorais apresentam positividade para este marcador.

Em ordem de frequiência, as metástases orbitárias em adultos são causadas por tumor maligno primário de mama (42\%), pulmão $(11 \%)$, próstata $(8,3 \%)$, melanoma $(5,2 \%)$ e trato gastrointestinal $(4,4 \%)^{(1-2,5)}$.

O melhor marcador sorológico do HCC disponível comercialmente é a alfa-fetoproteína (AFP) ${ }^{(1)}$. Existe uma forte correlação entre o tamanho do tumor e os níveis de AFP, porém em nódulos menores que $2 \mathrm{~cm}$, a AFP é normal em $80 \%$ dos $\operatorname{casos}^{(1-2)}$, como foi observado neste caso.

Em pacientes hepatopatas crônicos preconiza-se a realização de um estudo ultra-sonográfico abdominal e AFP seriadas a cada 6 meses para diagnóstico precoce de neoplasias hepáticas. Entretanto, nestes casos, o diagnóstico é confirmado através da biópsia da lesão primária ou metastática ${ }^{(1-2)}$.

As possibilidades terapêuticas do HCC consiste em ressecção cirúrgica de tumores pequenos, que pode ser curativa, associada a radioterapia externa ou quimioterapia sistêmica. Outras opções terapêuticas são quimioterapia intra-arterial, embolização tumoral e transplante hepático ${ }^{(1-2)}$.

O prognóstico do HCC quando existe metástases à distância é ruim, devido à rápida progressão da lesão primária e de múltiplas metástases, mesmo com tratamento paliativo ${ }^{(1-2)}$.

Neste caso, o diagnóstico foi confirmado pelo estudo anátomo-patológico. O paciente foi estadiado como T4 devido às metástases à distância (órbita).

Uma revisão na literatura demonstrou a existência de poucos casos de metástases orbitárias de HCC. Kim et al. ${ }^{\left({ }^{8}\right)}$ relataram um caso de metástase para a órbita de $\mathrm{HCC}$ em uma paciente com hepatite B e AFP elevada. Lubin et al. ${ }^{(6)}$ descreveram um caso de metástases orbitária e óssea em um paciente cirrótico. Outros dois relatos apresentam associação de metástases orbitárias e cerebrais ${ }^{(4,9)}$. Apenas Tranfa et al.$^{(5)}$ descreveram um caso de HCC com metástase inicial somente orbitária, sem fatores de risco associados e com diagnóstico anátomo-patológico confirmado.

Este caso é raro não apenas pela lesão metastática exclusivamente orbitária confirmada pelo estudo anatomopatológico, como também pela ausência de fatores de risco associados.

\section{ABSTRACT}

Orbital metastasis of hepatocellular carcinoma is rare. We report an unusual orbital metastatic lesion as the only finding in a case of hepatocellular carcinoma. A 57-year-old man presented with a 6-month history of orbital painful right orbital mass, associated with proptosis. Computed tomography of the orbits showed an orbital soft tissue mass leading to bone erosion and intracranial invasion. Computed tomography of the adbomen showed a focal perfusion abnormality in the left lobe of the liver. Incisional biopsy was performed and the histopathologic examination of the specimen confirmed the diagnosis. The patient died 15 months after the initial presentation. Coments: This is a rare case of orbital metastasis of hepatocellular carcinoma. There was no another metastatic lesion and the patient reported only ophthalmological symptoms.

Keywords: Neoplasm metastasis; Orbital neoplasms/secondary; Carcinoma, hepatocellular; Liver neoplasms [Publication type]

\section{REFERÊNCIAS}

1. Forones NM, Trivino T. Hepatocarcinoma. In: Prado FC, Ramos J, Valle JR. Atualização terapêutica. 19 $9^{\underline{a}}$ ed. Rio de Janeiro: Artes Médicas; 1999. Seção IV, p.507-8.

2. Friedman LS. Diseases of the liver In: Tierney LM, McPhee SJ, Papadakis MA. Current medical diagnosis \& treatment. $45^{\text {th }}$ ed. New York: McGrawHill Medical; 2003. Chapter 15, p.652-83.

3. Henderson JW. Orbital tumors. Philadelphia: Saunders; 1973. p.474-94.

4. Wakisaka S, Tashiro M, Nakano S, Kita T, Kisanuki H, Kinoshita K. Intracranial and orbital metastasis of hepatocellular carcinoma: report of two cases. Neurosurgery. 1990;26(5):863-6.p

5. Tranfa F, Cennamo G, Rosa N, De Rosa G, Boscaino A, Bonavolontà G. An unusual orbital lesion: hepatoma metastatic to the orbit. Ophthalmologica. 1994; 208(6):329-32.

6. Lubin JR, Grove AS Jr, Zakov ZN, Albert DM. Hepatoma metastatic to the orbit. Am J Ophthalmol. 1980;89(2):268-73.

7. Romanas MM, Cherian R, McGregor DH, Wu Y, May CL, Baranda JC. Hepatocellular carcinoma diagnosed by fine-needle aspiration of the parotid gland. Diagn Cytopathol. 2004;30(6):401-5.

8. Kim IT, Na SC, Jung BY. Hepatocellular carcinoma metastatic to the orbit. Korean J Ophthalmol. 2000;14(2):97-102.

9. Loo KT, Tsui WM, Chung KH, Ho LC, Tang SK, Tse CH. Hepatocellular carcinoma metastasizing to the brain and orbit: report of three cases. Pathology. 1994;26(2):119-22. 